\title{
Effect of Short-Term Whole-Body Vibration Training on Metabolic Risk Factors, Inflammatory Markers, and Arterial Stiffness
}

\author{
Tsuneo Watanabe, Tamotsu Yabumoto, Sohee Shin, Bateer Shi, Toshio Matsuoka \\ Department of Sports Medicine and Sports Science, Gifu University Graduate School of Medicine, Gifu, Japan \\ Email: tsuneo w@gifu-u.ac.jp
}

Received 23 January 2014; revised 7 March 2014; accepted 21 March 2014

Copyright (C) 2014 by authors and Scientific Research Publishing Inc. This work is licensed under the Creative Commons Attribution International License (CC BY). http://creativecommons.org/licenses/by/4.0/

(c) (i) Open Access

\section{Abstract}

To investigate the effect of aerobic exercise combined with whole-body vibration (WBV) training on metabolic syndrome risk, and inflammatory markers, and to compare its effects on arterial stiffness and several blood parameters related to metabolic syndrome with those of aerobic training alone. Thirty healthy participants were divided into 2 groups matched for age and body mass index (BMI). Fifteen subjects were assigned to the WBV group (WBV + aerobic training) and the other 15 to the control group (aerobic training only). All participants performed a treadmill exercise 3 days/week for 4 weeks, for $50 \mathrm{~min}$ after $30 \mathrm{~min}$ rest period. Exercise was performed at a heart rate (HR) corresponding to $60 \%$ of the $\mathrm{HR}$ at each individual's maximum oxygen uptake $\left(\mathrm{VO}_{2 \max }\right)$. Body weight and $\mathrm{BMI}$ after training were significantly lower than those before training in the WBV group $(P<0.01)$. Concerning arterial stiffness, the pulse-wave velocity after training was significantly lower than that before training in the WBV group $(1048.7 \pm 105.6$ vs. 1008.4 \pm 101.1 $\mathrm{cm} / \mathrm{s}, P<0.05$ ). Meanwhile, the $\mathrm{VO}_{2 \max }$ after training was significantly higher than that before training in the WBV group $\left(47.7 \pm 12.8\right.$ vs. $\left.51.4 \pm 14.1 \mathrm{~mL} \cdot \mathrm{min}^{-1} \cdot \mathrm{kg}^{-1}, P<0.01\right)$. However, no significant differences were observed in any parameters before and after training in the control group. Our results suggest that aerobic exercise combined with WBV training may be effective in reducing arterial stiffness and improving cardiorespiratory fitness than aerobic training alone.

\section{Keywords}

Whole-Body Vibration; Metabolic Syndrome; Arterial Stiffness; Pulse-Wave Velocity; Maximum Oxygen Uptake 


\section{Introduction}

Metabolic syndrome (MS) is a complex of symptoms including obesity, hyperglycemia, decreased high-density lipoprotein (HDL) levels, increased triglyceride (TG) levels, and high blood pressure, and also comprises risk factors for heart disease and other health problems such as diabetes and stroke [1]. The associations and clustering of these factors have been known for decades. Furthermore, MS has been clearly demonstrated to be common and have an increasing prevalence worldwide, which is largely associated with an increase in obesity and sedentary lifestyles [2]. Moreover, different regions have been found to have individual clusters of epidemic risk factors [3]. Thus, MS is currently in the spotlight as both a public health and a clinical problem.

Physical activity is well known to reduce the risk of cardiovascular disease in persons with established disorders such as obesity, hypertension, diabetes mellitus, and hyperlipidemia [4]-[7]. Increasing attention has been paid to whole-body vibration (WBV) training and its use as an alternative form of resistance training. WBV is implemented through the use of a vibrating platform on which exercise can be performed. The engines underneath the platform generate vibrations that are transmitted to the person standing, sitting, or lying on the machine. Several studies have recently reported the effect of WBV training on physical fitness in nonathletes, including its ability to reduce body weight or arterial stiffness [8]-[10]. Moreover, increasing evidence suggests that chronic subclinical inflammation is a part of MS. The measurement of inflammatory markers such as Creactive protein (CRP) has been proposed as a method to assess cardiovascular risk. However, few studies have examined the effect of WBV training on anti-inflammatory using CRP. Here, we aimed to investigate the effect of WBV training on MS risk, inflammatory marker, and arterial stiffness.

\section{Methods}

\subsection{Subjects}

Thirty healthy volunteers (age: $26.2 \pm 7.9$ years, height: $168.4 \pm 7.7 \mathrm{~cm}$, weight: $62.9 \pm 10.4 \mathrm{~kg}$, body mass index [BMI]: $22.1 \pm 2.6 \mathrm{~kg} / \mathrm{m}^{2}$ ) with no history of cardiopulmonary disease and no pathogenic conditions affecting the musculoskeletal or neuromuscular system were included in this study. Before the study, all subjects underwent a medical examination including electrocardiography and echocardiography at rest. In all 30 subjects, spiroergometry was performed to determine the maximum oxygen uptake $\left(\mathrm{VO}_{2 \max }\right)$ during exercise and to determine the study training intensity, which was set at a heart rate (HR) corresponding to $60 \%$ of that at $\mathrm{VO}_{2 \max }$. Participants were asked to continue with their normal prestudy diet. All subjects provided informed consent as required by the university institutional review board that approved the study.

\subsection{Protocol}

The subjects were divided into 2 groups that were matched for age and BMI. Fifteen subjects were assigned to the WBV group (WBV + aerobic training) and the other 15 were assigned to the control group (aerobic training only).

Experiments were conducted in a controlled environment with the temperature maintained at $25^{\circ} \mathrm{C}$. All participants performed a treadmill exercise 3 days/week for 4 weeks, for 50 min (including a 5-min warm-up and a 5-min cool-down) after a 30-min rest period. Exercise was performed at an $\mathrm{HR}$ corresponding to $60 \%$ of the HR at each individual's $\mathrm{VO}_{2 \max }$. During the exercise session, $\mathrm{HR}$ was monitored to ensure that the exercise intensity never exceeded $60 \%$ of the maximum HR. The WBV group performed the WBV training and the aerobic training on the same day. A training was performed on a WBV platform (Power Plate, Badhoevendorp, The Netherlands). A training protocol was designed with a gradual increase in frequency (30 - $35-40 \mathrm{~Hz}$ ), exercise time (30 - 45 s), and number of static exercises (10 - 12 exercises). The amplitude of each session was always low. The resting time between exercises always equaled the exercise time. The mean total exercise time per session was 13.6 min. Exercises were chosen to train all major muscle groups. A training session typically included: squats, deep squats, wide stance squats, calf raises, lunges, push-ups, triceps dips, biceps curls, abdominal crunches, and pelvic bridges. Laboratory parameters were determined at the beginning and end of the 4-week trial; height, body weight, body fat, and blood pressure were measured using a regular medical scale.

\subsection{Data Collection}

1) Anthropometric measurements. Anthropometric parameters were measured by trained staff, with the par- 
ticipants barefoot and in their underwear. Waist circumference was measured to the nearest $0.1 \mathrm{~cm}$ by using a vinyl tape measure, at the narrowest circumference between the lowest ribs and iliac crests while the subject was standing. The height was measured using an analog-type stadiometer to the nearest $0.1 \mathrm{~cm}$. Body weight and body fat were measured to the nearest $0.1 \mathrm{~kg}$ by using a BC-118D body composition analyzer (TANITA Co., Tokyo, Japan). Percentage body fat was evaluated by means of electrical impedance analysis. BMI was calculated as body weight $(\mathrm{kg}) /$ height $\left(\mathrm{m}^{2}\right)$.

2) Blood sampling. Subjects were asked to fast from 11:00 p.m. the evening before visiting the laboratory. A fasting blood sample was taken at noon on the day of the experiment from an antecubital vein, with the subject in a sitting position. The study parameters included total cholesterol (TC), HDL cholesterol, low-density lipoprotein (LDL) cholesterol, TG, fasting glucose, fasting insulin, C-reactive protein (CRP), and Interleukin (IL)-6. TC was analyzed using an enzymatic method; HDL and LDL were analyzed using direct methods; and TG was analyzed by elimination of free glycerol interference with a colorimetric enzymatic assay, using a JCA-BM6070 automated biochemical analyzer (JEOL Ltd., Tokyo, Japan). Insulin was measured using a chromogenic assay with a LUMIPULSE G1200 auto-immunoanalyzer (Fujirebio Inc., Tokyo, Japan). Glucose was measured using an enzyme assay with the GA09 system (A \& T Co., Yokohama, Japan). CRP was analyzed using nephelometry with the JCA-BM6070 automated biochemical analyzer (JEOL Ltd., Tokyo, Japan), whereas IL-6 was analyzed using enzyme-linked immunosorbent assay (eBioscience Inc., San Diego, CA, USA). The intra-assay coefficients of variation were $<3.0 \%$ for TC, TG, and CRP; $<5.0 \%$ for HDL, LDL, and glucose; and $<10 \%$ for insulin and IL-6.

3) Measurement of $\mathrm{VO}_{2 \max }$. Oxygen consumption was measured using a $\mathrm{V}_{\max } 29$ analyzer (CareFusion Co., Yorba Linda, CA, USA). $\mathrm{VO}_{2 \max }$ was measured using the modified Astrand protocol [11], in which participants ran at $70 \% \mathrm{HR}_{\max }$ for 3-min and the gradient was then increased by $2 \%$ every 2 min until the subject was exhausted. Although the use of this protocol and constant speed is associated with a slight risk of falling while running, it allows accurate measurement of $\mathrm{VO}_{2 \max }$ within a short time. $\mathrm{VO}_{2}$ was recorded continuously using a "breath-by-breath" method, and HR was measured simultaneously.

4) Measurement of arterial stiffness. As measures of arterial stiffeness, ankle-brachial pulse-wave velocity (PWV) and ankle-brachial index (ABI) were evaluated noninvasively with subjects in the supine position, using a form-I automated PWV/ABI analyzer (Colin Co. Ltd., Komaki, Japan) attached to the 4 limbs [12].

\subsection{Statistical Analysis}

Results are presented as mean $\pm \mathrm{SD}$, and statistical significance was set at $P<0.05$. The adequacy of the sample size was justified with power analysis calculations performed using $G^{*}$ power 3.1 (G*Power; Erdfelder, Faul, \& Buchner, 1996). To evaluate differences in values before and after intervention, a paired $t$-test was used. IBM SPSS Statistics 19.0 (IBM SPSS, Chicago, IL, USA) was used for the statistical calculations.

\section{Results}

The demographic and physiological baseline characteristics for both training groups are given in Table 1. Before training, no significant differences in any of the values were identified between the groups, with the exception of sex. Measurements of anthropometric, blood pressure, and blood parameters according to group as well as before and after training are shown in Table 2. Body weight and BMI after training were significantly lower than those before training in the WBV group (body weight: $59.3 \pm 7.7$ vs. $58.6 \pm 7.7 \mathrm{~kg}, P<0.01$; BMI: $21.5 \pm 1.9$ vs. $21.3 \pm 1.9 \%, P<0.01$ ). Concerning arterial stiffness, PWV after training was significantly lower than that before training in the WBV group (1048.7 \pm 105.6 vs. $1008.4 \pm 101.1 \mathrm{~cm} / \mathrm{s}, P<0.05$, Figure 1). Meanwhile, the $\mathrm{VO}_{2 \max }$ after training was significantly higher than that before training in the WBV group (47.7 \pm 12.8 vs. $51.4 \pm$ $14.1 \mathrm{~mL} \cdot \mathrm{min}^{-1} \mathrm{~kg}^{-1}, P<0.01$, Figure 2). However, no significant differences were observed in any blood parameters before and after training in both the control and WBV groups.

\section{Discussion}

The present study investigates the effect of short-term WBV training on metabolic risk, inflammatory markers, and arterial stiffness in healthy subjects. Changes in arteriosclerosis markers, such as decreased PWV and a significant increase in $\mathrm{VO}_{2 \max }$ levels, were observed in subjects who performed WBV training exercises. Our re- 
Table 1. Characteristics of Subjects.

\begin{tabular}{cccc}
\hline Parameters & Control group & WBV group & $P$-value \\
\hline Sex (male/female) & $12 / 3$ & $6 / 9$ & 0.030 \\
Age (years) & $25.7 \pm 8.2$ & $25.7 \pm 8.1$ & 0.738 \\
Height (cm) & $171.5 \pm 8.2$ & $165.8 \pm 7.2$ & 0.063 \\
Weight (kg) & $66.3 \pm 11.1$ & $59.3 \pm 7.7$ & 0.051 \\
BMI (kg/m $\left.{ }^{2}\right)$ & $22.5 \pm 3.1$ & $21.5 \pm 1.9$ & 0.212 \\
\% Body fat (\%) & $21.5 \pm 7.9$ & $25.3 \pm 8.2$ & 0.232 \\
\hline
\end{tabular}

BMI: body mass index.

Table 2. Comparison of several parameters before and after training.

\begin{tabular}{|c|c|c|c|c|c|c|}
\hline \multirow{2}{*}{ Variables } & \multicolumn{3}{|c|}{ Control $(\mathrm{n}=15)$} & \multicolumn{3}{|c|}{ WBV $(n=15)$} \\
\hline & Before & After & $P$-value & Before & After & $P$-value \\
\hline \multicolumn{7}{|c|}{ Anthropometric measurements } \\
\hline Weight (kg) & $66.3 \pm 11.1$ & $65.9 \pm 10.9$ & 0.129 & $59.3 \pm 7.7$ & $58.6 \pm 7.7$ & 0.006 \\
\hline $\operatorname{BMI}\left(\mathrm{kg} / \mathrm{m}^{2}\right)$ & $22.5 \pm 3.1$ & $22.4 \pm 3.1$ & 0.096 & $21.5 \pm 1.9$ & $21.3 \pm 1.9$ & 0.002 \\
\hline \% Body fat (\%) & $21.5 \pm 7.9$ & $21.8 \pm 8.0$ & 0.472 & $25.3 \pm 8.2$ & $25.1 \pm 8.2$ & 0.667 \\
\hline Waist (cm) & $78.4 \pm 10.3$ & $78.0 \pm 9.2$ & 0.475 & $75.4 \pm 7.3$ & $75.2 \pm 7.4$ & 0.401 \\
\hline \multicolumn{7}{|l|}{ Blood pressure } \\
\hline SBP (mmHg) & $122.2 \pm 3.8$ & $120.1 \pm 8.2$ & 0.352 & $112.8 \pm 7.5$ & $116.2 \pm 10.7$ & 0.193 \\
\hline DBP (mmHg) & $70.6 \pm 8.4$ & $68.5 \pm 6.7$ & 0.392 & $66.9 \pm 7.9$ & $65.4 \pm 3.9$ & 0.506 \\
\hline \multicolumn{7}{|l|}{ Blood tests } \\
\hline Glucose (mg/dL) & $91.5 \pm 9.9$ & $88.0 \pm 10.1$ & 0.107 & $96.2 \pm 7.6$ & $92.5 \pm 7.5$ & 0.104 \\
\hline Insulin $(u \mathrm{U} / \mathrm{mL})$ & $4.9 \pm 2.2$ & $5.2 \pm 2.9$ & 0.719 & $9.6 \pm 6.9$ & $9.4 \pm 8.4$ & 0.960 \\
\hline TG (mg/dL) & $87.4 \pm 49.8$ & $76.4 \pm 26.8$ & 0.378 & $66.6 \pm 32.4$ & $70.3 \pm 50.5$ & 0.718 \\
\hline $\mathrm{TC}(\mathrm{mg} / \mathrm{dL})$ & $177.6 \pm 25.8$ & $178.9 \pm 31.3$ & 0.747 & $167.3 \pm 21.5$ & $167.1 \pm 20.1$ & 0.958 \\
\hline HDL (mg/dL) & $59.2 \pm 13.1$ & $61.5 \pm 11.0$ & 0.962 & $58.9 \pm 10.5$ & $59.3 \pm 11.6$ & 0.839 \\
\hline LDL (mg/dL) & $100.8 \pm 29.3$ & $100.9 \pm 33.6$ & 0.962 & $95.2 \pm 24.2$ & $94.6 \pm 20.8$ & 0.847 \\
\hline CRP (mg/dL) & $0.04 \pm 0.04$ & $0.05 \pm 0.09$ & 0.758 & $0.03 \pm 0.02$ & $0.03 \pm 0.02$ & 0.655 \\
\hline IL-6 (pg/mL) & $2.4 \pm 3.4$ & $9.3 \pm 16.4$ & 0.225 & $36.2 \pm 57.8$ & $50.3 \pm 68.5$ & 0.493 \\
\hline
\end{tabular}

BMI, body mass index; SBP, systolic blood pressure; SDP, diastolic blood pressure; TG, triglycerides; TC, total cholesterol; HDL, high density lipoprotein cholesterol; LDL, low-density lipoprotein cholesterol; CRP, C-reactive protein; IL-6, Interleukin-6.

sults suggest that short-term aerobic exercise with WBV training is associated with the prevention of both obesity as well as arteriosclerosis.

The development of atherosclerosis increases the incidence of cardiovascular events such as heart attack or stroke. A study by Hirai et al. [13] showed strong associations between aortic stiffness and the degree of coronary artery disease, and similar associations have been reported between PWV and independently assessed cardiovascular risk scores [14]. PWV is generally assessed by measuring the time that the pulse wave takes to tra- 


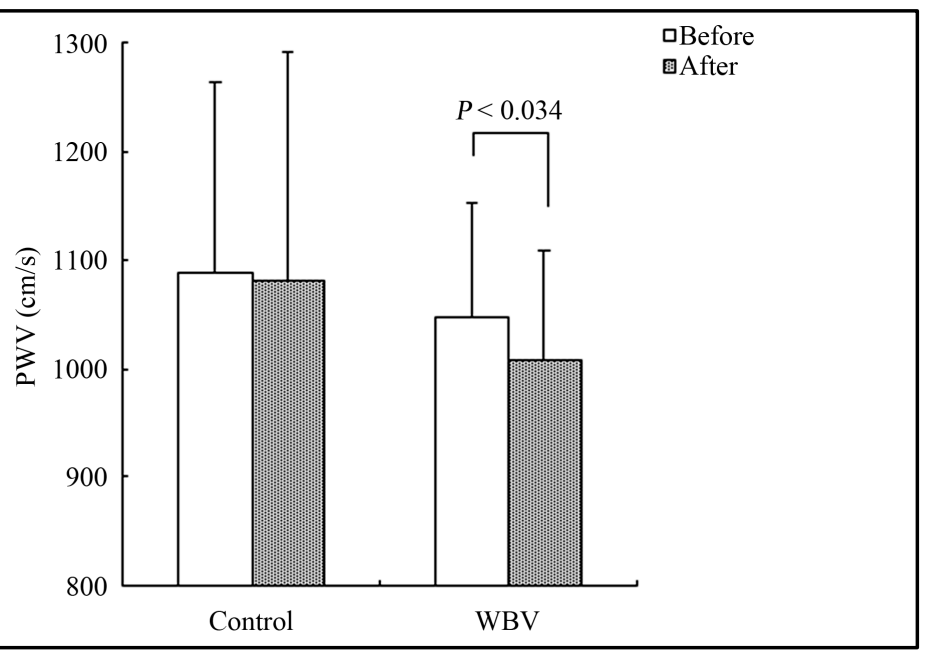

Figure 1. Change in the pulse-wave velocity levels in the control and WBV group.

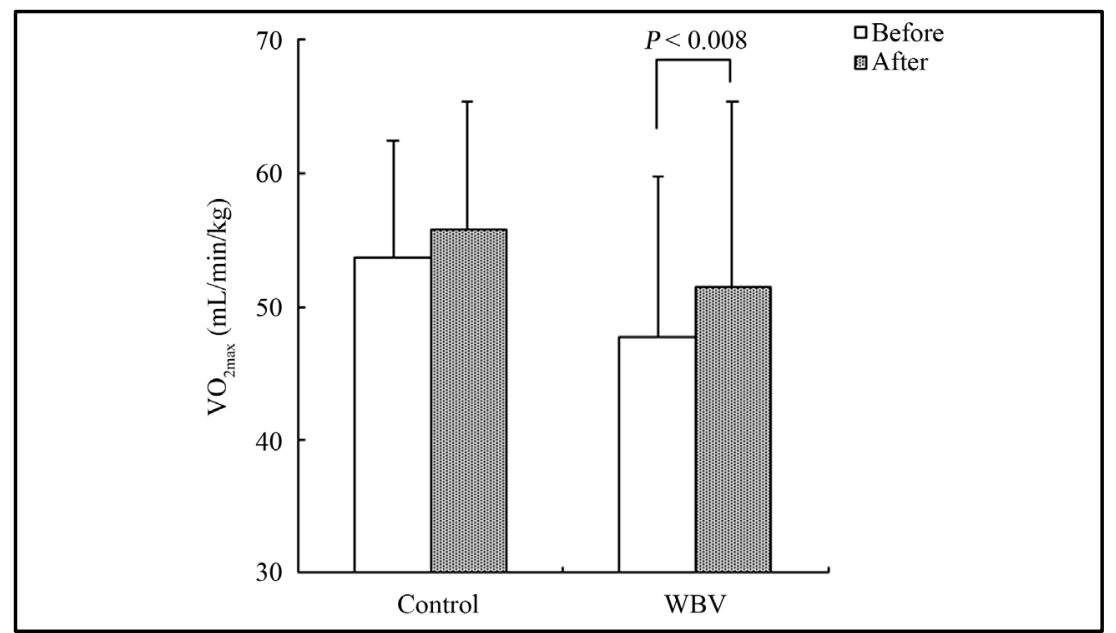

Figure 2. Change in mamimum oxygen uptake levels in the control and WBV group.

vel a given distance along the blood vessel and can provide an objective evaluation of atherosclerosis. Miyaki et al. [15] demonstrated a significant reduction in PWV by lifestyle modification combined with WBV training in overweight and obese women; however, they mentioned that the mechanisms underlying the decrease in arterial stiffness after WBV training are unclear. Otsuki et al. [16] investigated the effects of vibration on arterial stiffness by measuring the PWV, and found that arterial stiffness was significantly reduced at 20 and 40 min post vibration compared with that in the same time series of no vibration. Meanwhile, Lythgo et al. [17] found that application of acute intermittent vibration resulted in a significantly increase in mean blood cell velocity of the femoral artery. Moreover, Maloney-Hinds et al. [18] found that when the forearm was passively vibrated on a vertical synchronous vibration platform at 30 and $50 \mathrm{~Hz}$ for $10 \mathrm{~min}$, skin blood flow increased significantly within 5 min, which remained elevated for 9 min post vibration. We also confirmed significant increases in posterior tibial artery blood flow after vibration in a preliminary study (unpublished data). The vascular endothelium is a single layer of cells lining all the blood vessels in the body, and over the past three decades, it has emerged as a key player in vascular growth, vasoregulation, and vasoprotection. During this period of discovery, it was found that the endothelium is essential for vasodilatation in response to increases in blood flow associated shear stress [19] [20]. Several vasodilators are released by the endothelium in response to shear stress, including nitric oxide and prostaglandins [21] [22]. Thus, we hypothesize that the effect of WBV training on arterial stiffness is due to the stimulation of the endothelium following the increase in shear stress induced by increased blood flow. 
The inflammatory component of atherogenesis has been increasingly recognized over the last decade. It is well known that MS leads to cardiovascular disease. Central obesity and insulin resistance are believed to represent the common underlying factors of the syndrome, which features a chronic low-grade inflammatory state [23]. Low-grade chronic inflammation is reflected by increased CRP concentration and increased levels of certain cytokines [24], and CRP measurement has been proposed as a method for assessing cardiovascular risk. Furthermore, the presence of an anti-inflammatory effect of exercise has been indicated by changes in the levels of cytokines such as TNF- $\alpha$ and IL-6, as noted in a review article by Petersen and Pedersen [25]. In another study of inflammatory parameters in WBV training, Miyaki et al. [15] demonstrated the elevation of plasma pentraxin 3 (PTX3) level, which confers cardioprotective and atheroprotective effects. PTX3 adjusts both proinflammatory and anti-inflammatory factors; to limit the excess activation of inflammation, which may result in the prevention of cardiovascular disease [26]. Although the present study investigated whether WBV training reduces inflammatory markers such as CRP and IL-6, the levels of these markers did not change significantly in both the control and WBV groups during the study period. Thus, the authors could not conclude that WBV training has an anti-inflammatory effect.

Among patients with diabetes mellitus, increased physical activity or cardiorespiratory endurance capability is associated with a substantially reduced risk for cardiovascular events [27]-[29]. A recent study demonstrated that higher levels of cardiorespiratory fitness protect against MS in older persons [30]. Kumagai et al. [31] reported that a high degree of cardiorespiratory fitness positively contributed to the low prevalence of MS in patients with impaired glucose tolerance and type 2 diabetes mellitus. $\mathrm{VO}_{2 \max }$ is commonly used in exercise testing to assess a person's endurance capacity. Lakka et al. [32] investigated the leisure time physical activity and cardiorespiratory fitness in subjects with MS, and found that the associations of $\mathrm{VO}_{2 \max }$ with the components of the MS were stronger than the effects of physical activity. They emphasized that metabolic disorders may be suppressed by regular physical activity and maintaining a high value of the $\mathrm{VO}_{2 \max }$. Concerning WBV training, Miyaki et al. [15] reported that peak oxygen uptake significantly increased a after 12-week lifestyle modification program with WBV training. Rittweger et al. [33] investigated the effect of WBV on oxygen uptake, and found a linear increase in oxygen uptake from frequencies of 18 - $34 \mathrm{~Hz}$. Our results indicate that the $\mathrm{VO}_{2 \max }$ significantly increased after 4 weeks of regular aerobic exercise with WBV training compared with that before training; therefore, WBV training improves of cardiorespiratory fitness.

The values of MS risk markers did not change, although body weight was significantly reduced after training in the WBV group. Moreover, reductions in lipid levels or in glucose metabolism parameters were not observed after training in either the control or the WBV training group. Several authors have investigated whether WBV training combined with caloric restriction can reduce MS-related markers [8] [34]. Viessers et al. [8] found significant changes in lipid metabolism parameters after intervention. So et al. [34] also showed significant decreases in TC, LDL, and TG levels after intervention. However, our results showed that blood parameters related MS were not significantly changed after training in both groups, thus suggesting that aerobic exercise with WBV training alone is inefficient in changing the lipid blood parameters during this period. Further studies with larger numbers of participants are needed to confirm these results.

Our study has several limitations. First, the subjects were healthy young persons; thus, the reduction of MS risk markers due to regular WBV training was not evaluated in patients with metabolic disorders. Second, the training duration under each environmental condition was only 4 weeks. Thus, such a short-term study does not provide sufficient information about the reduction in arterial stiffness and improvement of $\mathrm{VO}_{2 \max }$. Third, the lack of a WBV training alone group as a limitation should be mentioned. To validate our findings and to confirm whether WBV training reduces the levels of MS risk markers and/or reduces or prevents arteriosclerosis, further studies with a larger number of participants and a long-term study design are warranted.

\section{Conclusion}

In conclusion, we found a significant decrease in PWV and a significant increase in $\mathrm{VO}_{2 \max }$ levels in our WBV training group, suggesting that regular short-term aerobic exercise combined with WBV training might more effectively reduce arterial stiffness and improve cardiorespiratory fitness than aerobic training alone.

\section{Acknowledgements}

The support of the staff at the Protea Japan Corporation is gratefully acknowledged. Authors also gratefully 
thank Mr. T. Chiba for helpful training program advice in this study.

\section{References}

[1] Magliano, D.J., Shaw, J.E. and Zimmet, P.Z. (2006) How to Best Define the Metabolic Syndrome. Annals of Medicine, 38, 34-41. http://dx.doi.org/10.1080/07853890500300311

[2] Alberti, K.G., Eckel, R.H., Grundy, S.M., Zimmet, P.Z., Cleeman, J.I., Donato, K.A., et al. (2009) Harmonizing the Metabolic Syndrome: A Joint Interim Statement of the International Diabetes Federation Task Force on Epidemiology and Prevention; National Heart, Lung and Blood Institute; American Heart Association; World Heart Federation; International Atherosclerosis Society; and International Association for the Study of Obesity. Circulation, 120, 16401645. http://dx.doi.org/10.1161/CIRCULATIONAHA.109.192644

[3] Eberly, L.E., Prineas, R., Cohen, J.D., Vazquez, G., Zhi, X., Neaton, J.D., et al. (2006) Metabolic Syndrome: Risk Factor Distribution and 18-Year Mortality in the Multiple Risk Factor Intervention Trial. Diabetes Care, 29, 123-130. http://dx.doi.org/10.2337/diacare.29.01.06.dc05-1320

[4] Guo, W., Kawano, H., Piao, L., Itoh, N., Node, K. and Sato, T. (2011) Effects of Aerobic Exercise on Lipid Profiles and High Molecular Weight Adiponectin in Japanese Workers. Internal Medicine, 50, 389-395.

http://dx.doi.org/10.2169/internalmedicine.50.4380

[5] Becker-Grunig, T., Klose, H., Ehlken, N., Lichtblau, M., Nagel, C., Fischer, C., et al. (2013) Efficacy of Exercise Training in Pulmonary Arterial Hypertension Associated with Congenital Heart Disease. International Journal of Cardiology, 168, 375-381. http://dx.doi.org/10.1016/j.ijcard.2012.09.036

[6] Braz, N.F., Carneiro, M.V., Oliveira-Ferreira, F., Arrieiro, A.N., Amorim, F.T., Lima, M.M., et al. (2012) Influence of Aerobic Training on Cardiovascular and Metabolic Parameters in Elderly Hypertensive Women. International Journal of Preventive Medicine, 3, 652-659.

[7] Voulgari, C., Pagoni, S., Vinik, A. and Poirier, P. (2013) Exercise Improves Cardiac Autonomic Function in Obesity and Diabetes. Metabolism 62, 609-621. http://dx.doi.org/10.1016/j.metabol.2012.09.005

[8] Vissers, D., Verrijken, A., Mertens, I., Van Gils, C., Van de Sompel, A., Truijen, S., et al. (2010) Effect of Long-Term Whole Body Vibration Training on Visceral Adipose Tissue: A Preliminary Report. Obesity Facts, 3, 93-100. http://dx.doi.org/10.1016/j.metabol.2012.09.005

[9] Behboudi, L., Azarbayjani, M.A., Aghaalinejad, H. and Salavati, M. (2011) Effects of Aerobic Exercise and Whole Body Vibration on Glycaemia Control in Type 2 Diabetic Males. Asian Journal of Sports Medicine, 2, 83-90.

[10] Figueroa, A., Gil, R., Wong, A., Hooshmand, S., Park, S.Y., Vicil, F., et al. (2012) Whole-Body Vibration Training Reduces Arterial Stiffness, Blood Pressure and Sympathovagal Balance in Young Overweight/Obese Women. Hypertension Research, 35, 667-672. http://dx.doi.org/10.1038/hr.2012.15

[11] Pollock, M.L., Wilmore, J.H. and Fox, S.M. (1984) Exercise in Health and Disease: Evaluation and Prescription for Prevention and Rehabilitation. W.B. Saunders, Philadelphia.

[12] Yamashina, A., Tomiyama, H., Takeda, K., Tsuda, H., Arai, T., Hirose, K., et al. (2002) Validity, Reproducibility, and Clinical Significance of Noninvasive Brachial-Ankle Pulse Wave Velocity Measurement. Hypertension Research, 25, 359-364. http://dx.doi.org/10.1291/hypres.25.359

[13] Hirai, T., Sasayama, S., Kawasaki, T. and Yagi, S. (1989) Stiffness of Systemic Arteries in Patients with Myocardial Infarction: A Noninvasive Method to Predict Severity of Coronary Atherosclerosis. Circulation, 80, 78-86. http://dx.doi.org/10.1161/01.CIR.80.1.78

[14] Lehmann, E.D., Hopkins, K.D., Rawesh, A., Joseph, R.C., Kongola, K., Coppack, S. W., et al. (1998) Relation between Number of Cardiovascular Risk Factors/Events and Noninvasive Doppler Ultrasound Assessments of Aortic Compliance. Hypertension, 32, 565-569. http://dx.doi.org/10.1161/01.HYP.32.3.565

[15] Miyaki, A., Maeda, S., Choi, Y., Akazawa, N., Tanabe, Y., So, R., et al. (2012) The Addition of Whole-Body Vibration to a Lifestyle Modification on Arterial Stiffness in Overweight and Obese Women. Artery Research, 6, 85-91. http://dx.doi.org/10.1016/j.artres.2012.01.006

[16] Otsuki, T., Takanami, Y., Aoi, W., Kawai, Y., Ichikawa, H. and Yoshikawa, T. (2008) Arterial Stiffness Acutely Decreases after Whole-Body Vibration in Humans. Acta Physiologica, 194, 189-194. http://dx.doi.org/10.1111/j.1748-1716.2008.01869.x

[17] Lythgo, N., Eser, P., de Groot, P. and Galea, M. (2009) Whole-Body Vibration Dosage Alters Leg Blood Flow. Clinical Physiology and Functional Imaging, 29, 53-59. http://dx.doi.org/10.1111/j.1475-097X.2008.00834.X

[18] Maloney-Hinds, C., Petrofsky, J. S. and Zimmerman, G. (2008) The Effect of 30 Hz vs. 50 Hz Passive Vibration and Duration of Vibration on Skin Blood Flow in the Arm. Medical Science Monitor, 14, CR112-116.

[19] Smiesko, V., Kozik, J. and Dolezel, S. (1985) Role of Endothelium in the Control of Arterial Diameter by Blood Flow. 
Blood Vessels, 22, 247-251.

[20] Pohl, U., Holtz, J., Busse, R. and Bassenge, E. (1986) Crucial Role of Endothelium in the Vasodilator Response to Increased Flow in Vivo. Hypertension, 8, 37-44. http://dx.doi.org/10.1161/01.HYP.8.1.37

[21] Joannides, R., Haefeli, W.E., Linder, L., Richard, V., Bakkali, E. H, Thuillez, C., et al. (1995) Nitric Oxide Is Responsible for Flow-Dependent Dilatation of Human Peripheral Conduit Arteries in Vivo. Circulation, 91, 1314-1319. http://dx.doi.org/10.1161/01.CIR.91.5.1314

[22] Okahara, K., Sun, B. and Kambayashi, J. (1998) Upregulation of Prostacyclin Synthesis-Related Gene Expression by Shear Stress in Vascular Endothelial Cells. Arteriosclerosis, Thrombosis, and Vascular Biology, 18, 1922-1926. http://dx.doi.org/10.1161/01.ATV.18.12.1922

[23] Paoletti, R., Bolego, C., Poli, A. and Cignarella, A. (2006) Metabolic Syndrome, Inflammation and Atherosclerosis. Vascular Health and Risk Management, 2, 145-152. http://dx.doi.org/10.2147/vhrm.2006.2.2.145

[24] Ross, R. (1999) Atherosclerosis Is an Inflammatory Disease. American Heart Journal, 138, S419-S420. http://dx.doi.org/10.1016/S0002-8703(99)70266-8

[25] Petersen, A.M. and Pedersen, B.K. (2005) The Anti-Inflammatory Effect of Exercise. Journal of Applied Physiology, 98, 1154-1162. http://dx.doi.org/10.1152/japplphysiol.00164.2004

[26] Norata, G.D., Marchesi, P., Pulakazhi Venu, V.K., Pasqualini, F., Anselmo, A., Moalli, F., et al. (2009) Deficiency of the Long Pentraxin PTX3 Promotes Vascular Inflammation and Atherosclerosis. Circulation, 120, 699-708. http://dx.doi.org/10.1161/CIRCULATIONAHA.108.806547

[27] Wei, M., Gibbons, L.W., Kampert, J.B., Nichaman, M.Z. and Blair, S.N. (2000) Low Cardiorespiratory Fitness and Physical Inactivity as Predictors of Mortality in Men with Type 2 Diabetes. Annals of Internal Medicine, 132, 605-611. http://dx.doi.org/10.7326/0003-4819-132-8-200004180-00002

[28] Hu, F.B., Stampfer, M.J., Solomon, C., Liu, S., Colditz, G.A., Speizer, F.E., et al. (2001) Physical Activity and Risk for Cardiovascular Events in Diabetic Women. Annals of Internal Medicine, 134, 96-105. http://dx.doi.org/10.7326/0003-4819-134-2-200101160-00009

[29] Church, T.S., Cheng, Y.J., Earnest, C.P., Barlow, C.E., Gibbons, L.W., Priest, E.L., et al. (2004) Exercise Capacity and Body Composition as Predictors of Mortality among Men with Diabetes. Diabetes Care, 27, 83-88. http://dx.doi.org/10.2337/diacare.27.1.83

[30] Hendriksen, I.J. and Meeuwsen, T. (2003) The Effect of Intermittent Training in Hypobaric Hypoxia on Sea-Level Exercise: A Cross-Over Study in Humans. European Journal of Applied Physiology, 88, 396-403. http://dx.doi.org/10.1007/s00421-002-0708-Z

[31] Kumagai, S., Kai, Y., Nagano, M., Zou, B., Kishimoto, H. and Sasaki, H. (2005) Relative Contributions of Cardiorespiratory Fitness and Visceral Fat to Metabolic Syndrome in Patients with Diabetes Mellitus. Metabolic Syndrome and Related Disorders, 3, 213-220. http://dx.doi.org/10.1089/met.2005.3.213

[32] Lakka, T.A., Laaksonen, D.E., Lakka, H.M., Mannikko, N., Niskanen, L.K., Rauramaa, R., et al. (2003) Sedentary Lifestyle, Poor Cardiorespiratory Fitness, and the Metabolic Syndrome. Medicine \& Science in Sports \& Exercise, 35, 1279-1286. http://dx.doi.org/10.1249/01.MSS.0000079076.74931.9A

[33] Rittweger, J., Ehrig, J., Just, K., Mutschelknauss, M., Kirsch, K.A. and Felsenberg, D. (2002) Oxygen Uptake in Whole-Body Vibration Exercise: Influence of Vibration Frequency, Amplitude, and External Load. International Journal of Sports Medicine, 23, 428-432. http://dx.doi.org/10.1055/s-2002-33739

[34] So, R., Etoa, M., Tsujimoto, T. and Tanaka, K. (2013) Acceleration Training for Improvingphysical Fitness and Weight Loss Inobese Women. Obesity Research \& Clinical Practice (Epub Ahead of Print). http://dx.doi.org/10.1016/j.orcp.2013.03.002 